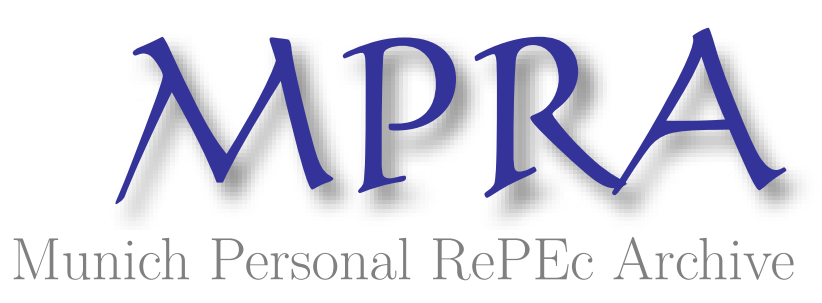

\title{
An Attempt to Control Tax Evasion
}

Rosato, Antonio

6 July 2006

Online at https://mpra.ub.uni-muenchen.de/15084/

MPRA Paper No. 15084, posted 09 May 2009 07:19 UTC 


\title{
An Attempt to Control Tax Evasion
}

\author{
Antonio Rosato*
}

7th May 2009

\begin{abstract}
The main object of this paper is the study of tax evasion from a theoretical point of view. We construct a simple two-player model in order to analyze the interaction between the taxpayer and the IRS and the main tool we use is game theory. We derive equilibrium solutions for the static game and we also provide some interesting comparative statics results. Then, we develop a dynamic game over an infinite number of periods. We show and comment two different kinds of long-run equlibria.
\end{abstract}

\section{Introduction}

$\triangleright$ The results provided by the 1998 Taxpayer Compliance Measurement Program (TCMP) show that only $53 \%$ of the population of the United States paid taxes correctly while $40 \%$ paid less and $7 \%$ paid even more than the due amount. ${ }^{1}$ If we accept that the declarations of this last $7 \%$ are the outcome of computational errors, and even assuming this is also true for a same percentage of the declarations that are below the due amount, we still have one third of American citizens cheating the Government. Indeed, the IRS has calculated that the tax gap increased by a fivefold between 1973 and 1992: from 22.7 to 95,3 billions dollars. $^{2}$

Clotfelter was the first, in 1983, to carry out an econometric study based on TCMP data in order to analyze how tax evasion react to changes in the penalty rate and the structure of detection. He estimated an elasticity coefficient of the declared income to the real one varying from 0.5 to 0.8 with respect to the different kinds of taxpayers.

*This work would not have been possible without the help and the supervision of Antonio Acconcia whom I gratefully acknowledge. I also have received numerous, very valuable comments from Marco Pagnozzi. Finally, I wish to thank Annalisa Scognamiglio and Paolo Zacchia. I alone am responsible for errors and omissions.

${ }^{1}$ The TCMP is a procedure, carried out by the Internal Revenue Service (IRS), for evaluating the degree of compliance of taxpayers.

${ }^{2}$ For a matter of completeness, it must be stated that in the same period we observe an increase in both tax evasion and overall GPA at the same time; therefore, the tax gap in 1992 is almost of the same percentage of the GPA, 17,3\%, as it was in 1973 . 
Beron, Tauchen and Witte (1992), working with the same data, showed that an increase of the probability of detection causes a significant increase in the declared income for some, but not all kinds of taxpayers.

Tax evasion is a big and unsolved issue to deal with, since its negative consequences arise in many different circumstances and the analysis could take several different ways. From a public economics point of view, tax evasion is a source of inequality since the tax system could become regressive instead of progressive (looking at the ex post income distribution). Moreover, evasion is basically a loss for the Government and this could have very negative effects in terms of public goods and services. Last but not least, tax evasion is often a source of social conflict between citizens and people become disaffected when Governments do not take the indispensable measures to deal with it.

In this paper we are not going to do an empirical analysis but we look instead at tax evasion from a theoretical point of view; we are concerned about the reasons that cause people to cheat as well as the instruments that the IRS could implement in order to confront this problematic situation.

Our starting point is the model of Allingham and Sandmo (1972), then modified by Yitzhaki (1974), and the model by Graetz, Reinganum and Wilde (1986). ${ }^{3}$ Excellent surveys on the topic are provided by Andreoni, Erard and Feinstein (1998) and Slemrod and Yitzhaki (2000).

As far as possible we perform a general analysis without introducing any particular binding hypotheses on the mathematics of the model unless it is compulsory or for the sake of clarity.

Following the main literature on the topic of tax evasion, we are concerned in personal income tax evasion; but the analysis as well as the conclusions are very general and they can be applied to any kind of tax.

The paper is organized as follows. Section 2 describes the economic model in a static framework and depicts the equilibrium, while Section 3 provides the results of the comparative statics analysis. In Section 4 we make some rather broad assumptions on the form of the functions and we show the equilibrium solutions for that kind of functions. In Section 5 we extend our model into a dynamic framework. Section 6 contains the solutions of this dynamic game.

Concluding comments are gathered in Section 7.

\section{The Model}

$\triangleright$ Following the same approach as Allingham and Sandmo (1972) and Graetz, Reinganum and Wilde (1986), we apply game theory and theory of choice under uncertainty to highlight the strategic interaction between the taxpayer and the IRS.

Basically, the starting point is the idea of using the same mental scheme of the Duopoly (or the Oligopoly broadly speaking) analysis in which the two

\footnotetext{
${ }^{3}$ Furthermore, we look also at the results obtained by Kolm (1973), Kaplow (1990), Cowell (1990), Feinstein (1991), Mayshar (1991) and Cremer and Ghavari (1994).
} 
agents play a simultaneous game maximizing their objective functions each agent taking his own decision without knowing the one of the other.

Therefore, the taxpayer chooses the fraction of his own income he wants to declare while the IRS chooses the probability of control; the game is solved if there exists a Pure Strategy Nash Equilibrium. Later on we will look at the problem of tax evasion in a dynamic framework developing a model in which the simple game is repeated over several periods.

The basic assumptions of the model, at least in its static version, are the following:

- the taxpayer is a rational self interested agent that maximizes his own utility;

- taxpayer's preferences can be represented by a Von Neumann - Morgenstern Expected Utility function that has the income level as its own argument;

- the taxpayer is aware of his own income, $Y$, and he chooses to declare only a certain fraction $\alpha Y$ with $0 \leq \alpha \leq 1$. We also make the standard hypothesis that the income is exogenous and is not observable a priori by the IRS;

- taxes are proportional to the declared income: $t \alpha Y ;^{4}$

- from the IRS's point of view, all taxpayers are identical; in short this means that the IRS does not have any elements for discriminating inside the pool of all potential evaders. This hypothesis is crucial because it allows us to say that for IRS choosing either to control a certain fraction, $p$, of all declarations or to control each declaration with probability $p$ gives exactly the same outcome; ${ }^{5}$

- all the controls carried out by the IRS are successful. When the taxpayer is discovered in cheating, he has to pay a proportional penalty fee on the undeclared fraction: $\pi(1-\alpha) Y$ with $\pi>t$. In anyway the IRS cannot ask more than $Y$.

- finally, the control is not costless and we assume a cost function $C(p)$ with $C^{\prime}(p)>0$ and $C^{\prime \prime}(p)>0$, where $p$ is the probability of control.

$\triangleright$ After having given the main hypotheses, we can look at the objective functions of both players.

If the taxpayer is not discovered, his final income will be $A \equiv Y(1-t \alpha)$, otherwise his final income will be $B \equiv Y[1-t \alpha-\pi(1-\alpha)]$.

\footnotetext{
${ }^{4}$ We could have chosen a more complicated form of tax but the general results of the model are unchanged.

${ }^{5}$ This hypothesis could appear unrealistic. But, even if we allow the IRS to be able to discriminate between two or more groups of taxpayers, we could still think of the IRS as playing a different game with each group and that in each group the taxpayers are supposed to be identical.
} 
The utility function of the taxpayer is given by $U(\cdot)$ that satisfies $U(0)=0$, $U^{\prime}>0$ and $U^{\prime \prime}<0$.

So, the agent will choose $\alpha$ in order to maximize:

$$
E(U)=(1-p) U(A)+p U(B)
$$

Taking the first order condition for (1) with respect to $\alpha$ we obtain:

$$
F O C:-t Y(1-p) U^{\prime}(A)+p Y(\pi-t) U^{\prime}(B)=0
$$

The second order condition for a maximum:

$$
S O C: D \equiv(t Y)^{2}(1-p) U^{\prime \prime}(A)+p Y^{2}(\pi-t)^{2} U^{\prime \prime}(B)<0
$$

is satisfied because the utility function $U(\cdot)$ is concave.

The IRS is risk neutral and chooses $p$ in order to maximize its expected revenue:

$$
E(R)=(1-p) t \alpha Y+p Y[t \alpha+\pi(1-\alpha)]-C(p)
$$

Taking the first order condition for (2) with respect to $p$ we obtain:

$$
F O C: \pi Y(1-\alpha)-C^{\prime}(p)=0
$$

The second order condition for a maximum

$$
S O C:-C^{\prime \prime}(p)<0
$$

is satisfied since we assumed that the cost function is convex.

Until now no assumptions on the form of the functions were made and the analysis is carried on with implicit functions; nevertheless, we can study the 
path of best-response functions that give rise to the following linear system of first order equations:

$$
\left\{\begin{array}{l}
-t Y(1-p) U^{\prime}(A)+p Y(\pi-t) U^{\prime}(B)=0 \\
\pi Y(1-\alpha)-C^{\prime}(p)=0
\end{array}\right.
$$

Even if we do not have a precise form for the best-response function $\alpha(p)$ and $p(\alpha)$, applying the Implicit Function Theorem we obtain:

$$
\frac{\partial \alpha}{\partial p}=-\frac{1}{D}\left[t Y U^{\prime}(A)+Y(\pi-t) U^{\prime}(B)\right]
$$

and

$$
\frac{\partial p}{\partial \alpha}=-\frac{\pi Y}{C^{\prime \prime}(p)}
$$

Due to previous assumptions on $D, U^{\prime}, \pi$ and $t$, expression (4) is positive. This means that $\alpha(p)$ is increasing, and, as we could expect, the bigger the probability of control is, the larger is the fraction of his own income the taxpayer will declare. Expression (5), instead, is negative and $p(\alpha)$ is decreasing: the more honest the declaration by the taxpayer is, the lower the probability of detection.

Even ignoring the analytical expressions of the two best-response function we know that one is increasing and the other is decreasing, and that, by assumption, they are both continuous; so, they must intersect each other in the plane $\{\alpha, p\}$.

\section{Comparative Statics Results}

$\triangleright$ In this section we show the comparative statics results. The analysis will be carried out as far as possible with implicit functions and we will introduce explicit functional forms only when it is necessary.

Let's recall the equilibrium strategies $\left(\alpha^{*}, p^{*}\right)$ obtained by solving the linear system (3). We can without any loss of generality from now on set $c(p)=\frac{c p^{2}}{2}$ with $c>1$, since this particular function verifies the assumptions $C^{\prime}(p)>0$ and $C^{\prime \prime}(p)>0$.

In order to study how variations of the parameters affect the equilibrium values of our endogenous variable we rewrite the first order conditions system in the following way 


$$
\left\{\begin{array}{l}
F^{1} \equiv Y\left[p(\pi-t) U^{\prime}(B)-t(1-p) U^{\prime}(A)\right] \equiv 0 \\
F^{2} \equiv \pi(1-\alpha) Y-c p \equiv 0
\end{array}\right.
$$

It is not trivial to show that the determinant of the Jacobian matrix ${ }^{6}$

$$
|J| \equiv\left|\begin{array}{cc}
F_{\alpha}^{1} & F_{p}^{1} \\
F_{\alpha}^{2} & F_{p}^{2}
\end{array}\right|=\left|\begin{array}{cc}
D & Y(\pi-t) U^{\prime}(B)+t U^{\prime}(A) \\
-\pi Y & -c
\end{array}\right|
$$

evaluated at $\left(\alpha^{*}, p^{*}\right)$ is strictly positive. So the $\frac{\partial \alpha}{\partial \pi}$ has the opposite sign with respect to the determinant

$$
\left|\begin{array}{cc}
F_{\pi}^{1} & F_{p}^{1} \\
F_{\pi}^{2} & F_{p}^{2}
\end{array}\right|=\left|\begin{array}{cc}
Y p\left[U^{\prime}(B)+(\pi-t) U^{\prime \prime}(B)(\alpha-1) Y\right] & Y(\pi-t) U^{\prime}(B)+t U^{\prime}(A) \\
Y(1-\alpha) & -c
\end{array}\right|
$$

Since this is negative, we can claim that $\frac{\partial \alpha}{\partial \pi}>0$. Similarly to the AllinghamSandmo model, an increased sanction forces the taxpayer to declare a higher percentage of his income.

The sign of the derivative of $p$ with respect to $\pi$ is opposite to the sign of the following determinant

$$
\left|\begin{array}{cc}
F_{\alpha}^{1} & F_{\pi}^{1} \\
F_{\alpha}^{2} & F_{\pi}^{2}
\end{array}\right|=\left|\begin{array}{cc}
D & Y p\left[U^{\prime}(B)+(\pi-t) U^{\prime \prime}(B)(\alpha-1) Y\right] \\
-\pi Y & Y(1-\alpha)
\end{array}\right|
$$

which is positive if

$$
\pi \leq t\left(1+Y^{2} \frac{(1-p) U^{\prime \prime}(A)}{U^{\prime \prime}(B)}\right)
$$

If condition (6) holds, we can claim that $\frac{\partial p}{\partial \pi}<0$; therefore, the higher the sanction is, the lower the probability of detection. This conclusion is totally consistent with the one of Becker (1968): that sanctions and control are substitute instruments because the Government is not able to increase both them at the same time and as much as it likes.

\footnotetext{
${ }^{6}$ The Jacobian is the vector of the first partial derivatives of a function. Since we consider more functions at the same time, we have a matrix with a number of rows equal to the number of functions and a number of columns equal to the number of variables.
} 
Unfortunately, if (6) does not hold, the effect that a variation of $\pi$ has on $p$ is ambiguous. But, (6) and the initial hypothesis $\pi>t$ mean that

$$
\left.\pi \in] t, t\left(1+Y^{2} \frac{(1-p) U^{\prime \prime}(A)}{U^{\prime \prime}(B)}\right)\right]
$$

In other words, the penalty fee must lie in a given range and this is what we observe in the real world. Indeed, if it is true that the penalty fee is bigger than taxes, it is even more true that in almost all countries law imposes constraints and upper bounds on the sanctions.

So, condition (6), as being realistic, can be embedded as one of our initial assumptions and in this way we eliminate the uncertainty on the sign of $\frac{\partial p}{\partial \pi}$.

With respect to the other parameters, tax fee and income, we cannot achieve unambiguous conclusions. Indeed, the $\frac{\partial \alpha}{\partial t}$ and the determinant

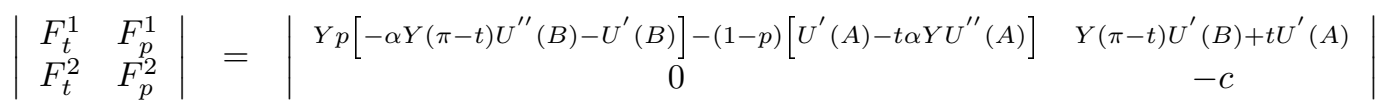

have opposite signes, but the latter is ambiguous because we cannot say anything about the sign of $F_{t}^{1}$ element .

The same argument applies also to $\frac{\partial p}{\partial t}$; actually, the sign of the determinant

$$
\left|\begin{array}{ll}
F_{\alpha}^{1} & F_{t}^{1} \\
F_{\alpha}^{2} & F_{t}^{2}
\end{array}\right|=\left|\begin{array}{cc}
D & Y p\left[-\alpha Y(\pi-t) U^{\prime \prime}(B)-U^{\prime}(B)\right]-(1-p)\left[U^{\prime}(A)-t \alpha Y U^{\prime \prime}(A)\right] \\
-\pi Y & 0
\end{array}\right|
$$

is not determined again because of $F_{t}^{1}$.

Comparative statics results related to changes in the taxpayer's income level are ambiguous too and we omit to proof them essentially for a matter of space; indeed, the explanation is quite similar: the presence of an ambiguous term makes it impossible to establish the sign of the determinant.

At this point in the discussion, we need to introduce some peculiar assumptions on the analytical form of the functions of our model; and we are going to do that in the next section.

\section{The solutions of the static model}

$\triangleright$ As pointed out in the previous section, the main complication is due to the utility function of the taxpayer.

Within the broad set of concave functions that could be chosen in order to represent the preferences of the agent, according to the assumptions of the model, we have selected the logarithmic one for a matter of simplicity as well for being in tune with the main literature on this field. 
We define $U(x)=\ln (1+x)$, where $x$ is the final income of the agent (including the taxes $)^{7}$ and we rewrite the two objective functions

$$
\begin{gathered}
E[U]=(1-p) \ln (1+Y-t \alpha Y)+p \ln (1+Y-t \alpha Y-\pi(1-\alpha) Y) \\
E[R]=(1-p) t \alpha Y+p(t \alpha Y+\pi(1-\alpha) Y)-\frac{c p^{2}}{2}
\end{gathered}
$$

$\triangleright$ Taking the first order condition with respect to $\alpha$ and $p$ for (7) and (8), respectively, we obtain an explicit form for system (3)

$$
\left\{\begin{array}{l}
\alpha=\frac{\frac{1}{Y}(\pi p-t)+t(\pi-1)+(1-t) p \pi}{t(\pi-t)} \\
p=\frac{\pi Y(1-\alpha)}{c}
\end{array}\right.
$$

$\triangleright$ It is easy to check that the best-response function of the IRS, $p(\alpha)$ decreases with the cost of detection $c$ and increases with the level of income $Y$ as well as the penalty fee $\pi$; furthermore, it does not depend on the tax level, $t$.

Taxpayer's best response, $\alpha(p)$ decreases with income and tax level but it increases with the penalty fee. Following our model's assumptions, tax evasion is a decreasing function of the sanction (and we had already found this result in Section 3 by employing implicit functions) but it increases with the income and taxes level. So, according to this simple model, evasion should be a "rich persons" phenomenon. This conclusion is consistent with the idea of a decreasing ArrowPratt absolute risk aversion measure and is also, in our opinion, very close to what we observe in the real world. ${ }^{8}$

By solving system (3) in its new explicit form, we get the unique Pure Strategy Nash Equilibrium of the game, that is given by the following strategies

$$
\alpha^{*}=\frac{c t\left(\pi-1-\frac{1}{Y}\right)+\pi^{2}[1+Y(1-t)]}{c t(\pi-t)+\pi^{2}[1+Y(1-t)]} \text { and } p^{*}=\frac{\pi t[1+Y(1-t)]}{c t(\pi-t)+\pi^{2}[1+Y(1-t)]}
$$

\footnotetext{
${ }^{7}$ We introduce a constant inside the logarithm in order to have the utility function welldefined also at zero: $U(0)=\ln (1)=0$.

${ }^{8}$ See Arrow (1970).
} 
Now we have to check if the above solutions satisfy two binding conditions. We need:

1. $\left(\alpha^{*}, p^{*}\right) \geq 0$

2. $\left(\alpha^{*}, p^{*}\right) \in[0,1]$

For $p^{*}$ the first condition is easily verified, while for $\alpha^{*}$ we need

$$
y^{2} \pi^{2}(1-t)+y\left[\pi^{2}-c t(1-\pi)\right]-c t \geq 0
$$

Expression (9) is verified for all values of $y$ such that ${ }^{9}$

$$
y \geq \frac{c t(1-\pi)-\pi^{2}+\sqrt{\left[\pi^{2}-c t(1-\pi)\right]^{2}+4 c t \pi^{2}(1-t)}}{2 \pi^{2}(1-t)}
$$

The second condition is a bit trickier. For $p^{*}$ it is very simple to check that since

$$
\pi Y t(1-t)+\pi t \leq \pi^{2} Y(1-t)+\pi^{2}
$$

it must also hold that

$$
\pi Y t(1-t)+\pi t<\pi^{2} Y(1-t)+\pi^{2}+c t(\pi-t)
$$

$\triangleright$ For $\alpha^{*}$ we require

$$
c t\left(\pi-1-\frac{1}{Y}\right)+\pi^{2}[1+Y(1-t)] \leq c t(\pi-t)+\pi^{2}[1+Y(1-t)]
$$

that, with simple mathematics, leads to

$$
t \leq 1+\frac{1}{Y}
$$

which is obviously satisfied since $t$ must be less than 1 .

We now can jump to the conclusion that our solutions lie inside the $(0,1)$ interval and that means that there are no corner solutions. From a more practical point of view, we are claiming that it is impossible for a taxpayer to be

\footnotetext{
${ }^{9}$ Although the mathematics could seem a bit tedious, after all we are just claiming that the taxpayer declares a positive amount if and only if his income is above a given threshold level. Indeed, this is very close to what we observe in all those countries where low income categories are usually excluded from paying taxes.
} 
"completely honest" $(\alpha=1)$ or "completely evader" $(\alpha=0)$; similarly, the IRS will always control a strictly positive fraction of the declarations but, it will never check all of them.

Comparative statics about the effect produced by a variation of $\pi$ over the equilibrium values $\left(\alpha^{*}, p^{*}\right)$ are consistent with the results we provided in previous section. ${ }^{10}$ For the tax fee $t$ it is easy to check that $\frac{\partial \alpha^{*}}{\partial t}<0$ and $\frac{\partial p^{*}}{\partial t}>0 .{ }^{11}$ The higher the tax fee is the lower will be the fraction of income reported by the taxpayer because the marginal benefit of evasion increases; on the other side, the IRS is going to check a higher percentage of declarations since the marginal benefit of detection increases as both tax fee and evasion become higher.

With respect to income, we have $\frac{\partial \alpha^{*}}{\partial Y}>0$ and $\frac{\partial p^{*}}{\partial Y}>0 .{ }^{12}$ Therefore, an increase in the income level results in both a higher percentage of the declared income and a higher probability of control. At a first glance, this could seem a bit bizarre since we previously showed that the taxpayer's best response function is decreasing with his own income; but, at the equilibrium the higher the income is the more honest the taxpayer will be since the IRS is going to increase the number of controls.

Finally, an increased control cost, provokes the results we expected: the number of controls diminishes while the evasion increases. Algebrically:

$$
\frac{\partial \alpha^{*}}{\partial c} \equiv \frac{t\left(\pi-1-\frac{1}{Y}\right) \cdot\left[c t(\pi-t)+\pi^{2}+\pi^{2} Y(1-t)\right]-t(\pi-t) \cdot\left[c t\left(\pi-1-\frac{1}{Y}\right)+\pi^{2}+\pi^{2} Y(1-t)\right]}{\left[c t(\pi-t)+\pi^{2}+\pi^{2} Y(1-t)\right]^{2}}>0
$$

and

$$
\frac{\partial p^{*}}{\partial c} \equiv \frac{-t(\pi-t)}{\left[c t(\pi-t)+\pi^{2}+\pi^{2} Y(1-t)\right]^{2}}<0
$$

\footnotetext{
${ }^{10} \mathrm{~A}$ variation of $\pi$ causes the numerator as well the denominator of $\alpha^{*}$ to increase; but, since the denominator grows slower than the numerator, we can state that $\frac{\partial \alpha^{*}}{\partial \pi}>0$.

${ }^{11}$ The two derivatives are:

$\frac{\partial \alpha^{*}}{\partial t} \equiv \frac{\left[c\left(\pi-1-\frac{1}{Y}\right)-\pi^{2} Y\right] \cdot\left[c t(\pi-t)+\pi^{2}+\pi^{2} Y(1-t)\right]-\left[c \pi-2 c t-\pi^{2} Y\right] \cdot\left[c t\left(\pi-1-\frac{1}{Y}\right)+\pi^{2}+\pi^{2} Y(1-t)\right]}{\left[c t(\pi-t)+\pi^{2}+\pi^{2} Y(1-t)\right]^{2}}<0$

and

$\frac{\partial p^{*}}{\partial t} \equiv \frac{\pi[\pi+Y(1-2 t)] \cdot\left[c t(\pi-t)+\pi^{2}+\pi^{2} Y(1-t)\right]-\left[c \pi-2 c t-\pi^{2} Y\right] \cdot[\pi Y t(1-t)+\pi t]}{\left[c t(\pi-t)+\pi^{2}+\pi^{2} Y(1-t)\right]^{2}}>0$

${ }^{12}$ The two derivatives are:

$\frac{\partial \alpha^{*}}{\partial Y} \equiv \frac{\left[\frac{1}{Y^{2}}+\pi^{2}(1-t)\right] \cdot\left[c t(\pi-t)+\pi^{2}+\pi^{2} Y(1-t)\right]-\pi^{2}(1-t) \cdot\left[c t\left(\pi-1-\frac{1}{Y}\right)+\pi^{2}+\pi^{2} Y(1-t)\right]}{\left[c t(\pi-t)+\pi^{2}+\pi^{2} Y(1-t)\right]^{2}}$

$\frac{\partial p^{*}}{\partial Y} \equiv \frac{\pi t(1-t) \cdot\left[c t(\pi-t)+\pi^{2}+\pi^{2} Y(1-t)\right]-\pi^{2}(1-t) \cdot[\pi Y t(1-t)+\pi t]}{\left[c t(\pi-t)+\pi^{2}+\pi^{2} Y(1-t)\right]^{2}}$
} 
We conclude this section by noting that these comparative statics results, once explicit hypotheses on functional forms have been introduced, are consistent with those of the literature on tax evasion as well as with the main features of the phenomenon we want to explain.

\section{The Dynamic Case}

$\triangleright$ The equilibrium we found in the previous section is a good result for economic textbooks but it is unlikely to find it in the real world. There are two features affecting tax evasion that we have not yet taken into account: in the real world, the IRS is subject to informational as well as technological constraints that make it unable to implement its optimal strategy; moreover, since the interaction is repeated at least once a year, both players can observe the strategy of the rival and modify their own optimal strategy.

In order to investigate how the interaction between the taxpayer and the IRS develops over time, we imply a partial-adjustment model and we define a policy rule for the IRS as the following one

$$
\triangle p_{t+1}=\beta\left(p^{*}-p_{t}\right)
$$

where $\triangle p_{t+1} \equiv p_{t+1}-p_{t}$ and $\beta \in(0,1)$ is a parameter.

Expression (10) can also be rewritten in the more familiar way

$$
p_{t+1}=(1-\beta) p_{t}+\beta p^{*}
$$

Expression (11) is a first order difference equation and states that the probability chosen by the IRS at time $t+1$ is a linear combination of the probability chosen at time $t$ and of the optimal probability $p^{*}$; once solved, this equation gives us the expression of $p$ for every possible value of $t$.

We assume the taxpayer is aware of this policy rule and at each time $t$ he chooses the $\alpha_{t}^{*}$ that maximizes his expected utility ${ }^{13}$

$$
E\left(U_{t}\right)=\left(1-p_{t}\right) U\left(Y-t \alpha_{t} Y\right)+p_{t}\left(Y-t \alpha_{t} Y-\pi\left(1-\alpha_{t}\right)\right)
$$

\footnotetext{
${ }^{13}$ Here we are making two important assumptions. Firstly, we state that the taxpayer knows the IRS's policy rules and this means that to some extent he is able to predict the probability of control which he will be subject to; this could seem a pretty strong assumption, but we believe that the IRS here is the weak side and the huge amount reached by tax evasion should confirm our point of view. Secondly, we are keeping all parameters $(Y, t, \pi)$ constant over time; this is not a too strong assumption and we make it for simplicity since the general implications of the model would not be affected if we allowed parameters to change over time.
} 
An equation like the (11) is defined autonomous if, like in our case, time does not appear as an explicit argument of the function. In this kind of model, we look for a particular kind of solution, the asymptotic or long-run solution, which has to be well defined.

Now, our first concern is to find an initial condition; roughly speaking: since our model lasts for infinite periods, $t \in\left[0,+\infty\left[\right.\right.$, how can the IRS choose $p_{0}$ ?

The answer is pretty easy. Indeed, at $t=0$ the game has not already started and no interaction has taken place; therefore, it is very realistic to assume that at $t=0$ the IRS randomly chooses $p_{0}=\overline{p_{0}}$. Furthermore, at the first stage we also have an initial condition for the taxpayer, $\alpha_{0}^{*}\left(p_{0}\right) .{ }^{14}$

This in mind, solutions still differ with respect to the meaning we want to assign to $p^{*}$, or, in different words, to the behavior of the IRS.

If the IRS is a sophisticated agent it is able to find out the optimal solution of the static model, $p^{*}=\frac{\pi t[1+Y(1-t)]}{c t(\pi-t)+\pi^{2}[1+Y(1-t)]}$, but for technological and/or informational constraints cannot implement it from the first stage and it has to correct its own strategy at each period. In this framework, we should think $p^{*}$ as a constant term: $p_{t}^{*}=p^{*} \forall t$.

The story complicates if we consider the IRS to be a non sophisticated agent; in this case, $p^{*}$ should not be considered as constant and we will have $p_{t}^{*}\left(\alpha_{t}^{*}\right) \neq p_{s}^{*}\left(\alpha_{s}^{*}\right)$ for $t \neq s$ with $p_{t}^{*}=\frac{\pi Y\left(1-\alpha_{t}\right)}{c}$ (which is the same best response function of the static model). ${ }^{15}$

\section{The Solutions of the Dynamic Model}

$\triangleright$ In this section we show the equilibrium strategy of the IRS. We first look at the sophisticated case and then at the non sophisticated one. The analysis carried out and the conclusions are significantly different.

A sophisticated IRS chooses randomly its strategy at $t=0$ and plays $p_{0}=\overline{p_{0}}$. Then, starting from period $t=1$ on, for each iteration it chooses a probability $p_{t}$ that is nothing more than a linear combination of $p_{t-1}$, the probability it played in the previous stage, and $p^{*}$, the optimal probability. Therefore, for the generic period $t$ we have

$$
p_{t}=(1-\beta) p_{t-1}+\beta p^{*}
$$

Using the Superposition Principle, we can write the solution of (12) as

$$
p_{t}=p_{t}^{c}+p_{t}^{p}
$$

\footnotetext{
${ }^{14}$ Once you chose an initial condition for $p_{0}$ you automatically obtain the initial condition for $\alpha_{0}$ without any loss of generality.

${ }^{15}$ The idea of a non sophisticated IRS is more realistic as well as more suited to undertake the Italian situation.
} 
where $p_{t}^{c}$ is the general solution of the homogeneous equation $p_{t}=(1-\beta) p_{t-1}$ and $p_{t}^{p}$ is a particular solution. The solution of a generic homogeneous equation $x_{t}=a x_{t-1}$ is given by $c a^{t}$ where $c$ is a constant. So, the general solution of (12) is given by

$$
p_{t}=p_{0}(1-\beta)^{t}+p_{t}^{p}
$$

A natural candidate for the particular solution is a constant solution or , in other words, a steady state solution. But we already have in our model a constant solution, and this is $p^{*} .{ }^{16}$

Therefore, the complete solution of (12) is:

$$
p_{t}=p_{0}(1-\beta)^{t}+p^{*}
$$

It is straightforward that as $t \rightarrow \infty$ the term $p_{0}(1-\beta)^{t}$ vanishes and the system converges to the steady state solution $p^{*}$ no matter what $p_{0}$ is. This means that no matter what the IRS chooses to do in the first stage, a stable equilibrium will be achieved and this is the same equilibrium of the static model. ${ }^{17}$

The duration of the adjustment towards the equilibrium depends on $\beta$ and on the difference term $\left(p_{0}-p^{*}\right)$. Moreover, also the paths of the adjustments are different for the two players:

- if $p_{0}<p^{*}$, the IRS increases period-by-period its controls while the taxpayer becomes each period more honest;

- if $p_{0}>p^{*}$, at $t=0$ the IRS is controlling too much; therefore, it has to reduce its level of control while the taxpayer will declare less.

The case of a non sophisticated IRS is different since it does not know the equilibrium level of control of the static game and its policy rule is

\footnotetext{
${ }^{16}$ This is not very surprising; if we have an equilibrium solution for the static model, it is quite obvious that the constant solution of the dynamic model will be the same as the one of the static model.

${ }^{17}$ Indeed, the taxpayer equilibrium strategy is again,$$
\alpha^{*}=\frac{c t\left(\pi-1-\frac{1}{Y}\right)+\pi^{2}[1+Y(1-t)]}{c t(\pi-t)+\pi^{2}[1+Y(1-t)]} .
$$

The interpretation is the following: if at a certain period $t$ the IRS plays the equilibrium strategy of the static game, the taxpayer cannot be better off than by playing the equilibrium strategy of the static game; and since this is a stable solution, they do not have any incentive to unilaterally change their strategy.
} 


$$
p_{t}=(1-\beta) p_{t-1}+\beta p_{t-1}^{*}
$$

The game starts again with $p_{0}=\overline{p_{0}}$, but its evolution is completely different. In this case, at each period $t>0$ the IRS plays a probability that is a linear combination of the probability chosen the previous period and of the probability it should have chosen the previous period. Therefore, the IRS suffers a huge informative gap: at the end of each period it can observe $\alpha_{t}^{*}$ but it is too late. It will use $\alpha_{t}^{*}$ to establish the probability $p_{t}^{*}\left(\alpha_{t}^{*}\right)$ that it should have played and this probability will contribute to fix $p_{t+1}$ and so on.

Again, we can rewrite (14) as

$$
p_{t}=p_{t}^{c}+p_{t}^{p}
$$

or more explicitly like

$$
p_{t}=p_{0}(1-\beta)^{t}+p_{t}^{p}
$$

The crucial point now is that the particular solution is not constant anymore. There are several forms of $p_{t}^{p}$ and some are easier than others; here we develop the forward-looking solution.

We rewrite (14) "backward" by recursive substitutions and we get:

$$
\begin{aligned}
& p_{t}=(1-\beta) p_{t-1}+\beta p_{t-1}^{*} \\
& =(1-\beta)^{2} p_{t-2}+(1-\beta) \beta p_{t-2}^{*}+\beta p_{t}^{*} \\
& =(1-\beta)^{3} p_{t-3}+(1-\beta)^{2} \beta p_{t-3}^{*}+(1-\beta) \beta p_{t-2}^{*}+\beta p_{t}^{*} \\
& =(1-\beta)^{4} p_{t-4}+(1-\beta)^{3} \beta p_{t-4}^{*}+(1-\beta)^{2} \beta p_{t-3}^{*}+(1-\beta) \beta p_{t-2}^{*}+\beta p_{t}^{*} \\
& \quad \cdot \\
& \quad \cdot \\
& \text { and so on...... }
\end{aligned}
$$

The solution of this equation is given by

$$
p_{t}=(1-\beta)^{t} p_{0}+\beta \sum_{s=1}^{t}\left[(1-\beta)^{s-1} p_{t-s}^{*}\right]
$$

Expression (15) tells us that the control at time $t$ is a function of the initial condition $p_{0}$ and of all the shocks from 0 to $t . .^{18}$

Again, as $t \rightarrow \infty$ the term $(1-\beta)^{t} p_{0}$ vanishes, but we have to check if $\lim _{t \rightarrow \infty} \beta \sum_{s=1}^{t}\left[(1-\beta)^{s-1} p_{t-s}^{*}\right]$ is well defined. Only if the argument inside the summation has an upper bound, it will converge to a finite value.

${ }^{18}$ We can interpret these shocks as the mistakes done by the IRS in the control level. 
Proof $\triangleright$ By definition $p \in[0,1]$, so the second term on the right-hand side of expression (15) has the following upper bound:

$$
\beta \sum_{s=1}^{t}\left[(1-\beta)^{s-1} p_{t-s}^{*}\right] \leq \beta \sum_{s=1}^{t}(1-\beta)^{s-1}
$$

The right hand side is the sum of a geometric progression with common ratio $(1-\beta)$ so we can rewrite it like

$$
\beta \cdot \frac{1-(1-\beta)^{t}}{1-(1-\beta)}
$$

and its value is 1 as $t \rightarrow \infty$.

Since at least one from the $p_{i}{ }^{\prime} s$ has to be strictly less than one we conclude that $\beta \sum_{s=1}^{t}\left[(1-\beta)^{s-1} p_{t-s}^{*}\right]<1$.

Therefore, since the forward-looking solution exists and it is well defined, the equilibrium is asymptotically stable.

Here we will not look at the backward-looking solution; anyway it is easy to check that when the system allows for a stable forward looking solution, the backward-looking one is not well defined (and viceversa).

\section{Conclusions}

$\triangleright$ Our simple model provides some results that can be easily compared with those of the literature in this domain and also gives simple suggestions on the policy measures that the IRS could carry out in order to face the growing emergency of tax evasion.

The taxpayer of this model is very close to the Allingham-Sandmo's one: he is a rational and self interested agent and his only scope is the maximization of his expected utility. In the $\mathrm{A} / \mathrm{S}$ model the taxpayer has to choose the optimal amount of evasion $(X)$; in ours, he chooses the percentage $(\alpha)$ of his income to declare: the two frameworks are basically identical. The main difference between our model and the $\mathrm{A} / \mathrm{S}$ one is that here the taxpayer is aware of the strategic role played by the IRS, while in the other one the agency is supposed exogenous.

In modeling the objective function of the taxpayer we chose to not use an "Yitzhaki penalty fee" because we believe that in the real world evasion is strongly affected by the taxes' level. The comparative statics results confirm this idea showing that tax evasion is positively correlated with taxes. The interpretation is straightforward: the higher the taxes are the bigger is the marginal benefit from evasion and for $t \rightarrow \pi$ the taxpayer will declare nothing.

The choice of a Public Agency that maximizes its own expected revenue rather than a Social Welfare Function reflects our aim of following a more positive and less normative approach and is based on the assumption that the 
Government too, as being driven by politicians, is a rational and self interested agent.

In the model of Graetz, Reinganum and Wilde, the cost of detection was constant and given exogenous; instead, we wanted to make this variable endogenous and dependent of the probability of control. This happened for two important reasons. From a theoretical point of view, we think that it is more realistic to assume the cost of the control to be a function of the level of control itself and this is why we chose an increasing and convex cost function. Moreover, from a more technical point of view, with a constant cost it could have been impossible to obtain a best response function $p(\alpha)$ continuous and differentiable; and we would have lost ourselves in the "either-or prediction". ${ }^{19}$

Another important feature of our model is that the marginal gain of detection does not depend on the taxes' level. This is not by chance, but we wanted it; we look for a "fair" IRS that audits all the taxpayers in the same way, no matter what is the tax rate.

For what concerns the solutions, the model by Graetz, Reinganum and Wilde allows for two Pure Strategy Nash Equilibria: one is an internal while the other is a corner solution. This last one, they explain, could arise if the control costs are so high that the IRS chooses to not control. In our model, there is only one Pure Strategy Nash Equilibrium and this is an internal solution. Indeed, the IRS when choosing the optimal control is totally aware of the cost since this is linked to the control probability. So, when it chooses the optimal probability it also chooses, at the same time, the cost it wants to pay.

From a policy point of view, an internal solution means that even if the IRS will never control all the declarations, it should be able to reduce tax evasion at an "acceptable" level; but, at the same time an internal solution also means that tax evasion will still persist. Therefore, the aim of the IRS should to minimize the extent of the problem. This could be achieved through the acquisition of a more efficient technology of detection, since we showed that the lower the cost of control is, the smaller the evasion.

By the Dynamic Model we tried to highlight what we believe is the central point of the topic: the taxpayer got, gets and will always get an informational advantage. There are different explanations for that :

- the taxpayer has a kind of first move advantage since the game is not truly simultaneous;

- moreover, the taxpayer can infer the strategies and techniques of the IRS by the public announcements of the Government and has a private information, his own income level, that is hidden to the IRS;

- last but not least, the game is not perfectly symmetric in the sense that each taxpayer plays his own game against one rival while the IRS plays against a potential infinite number of rivals.

\footnotetext{
${ }^{19}$ We could have eliminated the "either-or prediction" and still keep a constant cost by assuming risk aversion (or propension) for the IRS. But since all the literature considers the IRS as a neutral risk agent and since we agree on this point, we chose to follow the mainstream.
} 
There are several directions for future research. First, we could look for the existence of other Nash Equlibria in mixed strategies. Second, we could try to better understand the relationship between probability of detection, evasion and the tax level by endogenating the last one too; we could think of a model in which the IRS has to choose, at the same time, both the optimal probability of control and the optimal tax level. Finally, in some way we should take in account that some sources of income are not able to evade taxes while others are more likely to do it. 


\section{References}

[1] ACCONCIA, A. "Endogenous Corruption and Tax Evasion in a Dynamic Model." CSEF working paper 154 (2006).

[2] ACEMOGLU, D. AND VERDIER, T. "The Choice between Market Failures and Corruption." American Economic Review, Vol.90 (2000), pp. 194211.

[3] ALlinghaM, G.M. AND SANDMO, A. "Income Tax Evasion: A Theoretical Analysis." Journal of Public Economics, Vol.1 (1972), pp.323-338.

[4] ANDREONI, J., ERARD, B. AND FEINSTEIN, J. "Tax Compliance." Journal of Economic Literature, Vol.36 (1998), pp. 818-860.

[5] ARROW, K.J. "Essays in Theory of Risk-Bearing." Amsterdam, North Holland, 1970.

[6] BECKER, S.G. "Crime and Punishment: An Economic Approach." Journal of Political Economy, Vol.76 (1968), pp.169-217.

[7] BERON, K.J., TAUCHEN, H.V. AND WITTE, A.D. "The Effect of Audit and Socioeconomic Variables on Compliance." in "Why People Pay Taxes", Slemrod J., University of Michigan Press, 1992.

[8] CLOTfElteR, C.T. "Tax Evasion and Tax Rates: An Analysis of Individual Returns." The Review of Economics and Statistics, Vol.65 (1983) pp.363-373.

[9] COWELL, F. "Cheating the Government: The Economics of Tax Evasion." Cambridge, Mass.: MIT Press, 1990.

[10] CREMER, H. AND GAHVARI, F. "Tax Evasion, Concealment and the Optimal Linear Income Tax." Scandinavian Journal of Economics, Vol.60 (1994), pp.219-239.

[11] FEINSTEIN, J. "An Econometric Analysis of Income Tax Evasion and its Detection." RAND Journal of Economics, Vol.22 (1991), pp.14-35.

[12] FUENTE DE LA, A. "Mathematical Methods and Models for Economists." Cambridge: Cambridge University Press, 2000.

[13] GRAETZ, M.J., REINGANUM, J.F. AND WILDE, L.L. "The Tax Compliance Game: Toward an Interactive Theory of Law Enforcement." Journal of Law, Economics and Organization, Vol.2 (1986), pp.1-32.

[14] KAPLOW, L. "Optimal Taxation with Costly Enforcement and Evasion." Journal of Public Economics, Vol.43 (1990), pp.221-236.

[15] KOLM, S.C. "A Note on Optimum Tax Evasion." Journal of Public Economics, Vol.2 (1973), pp.265-270. 
[16] MAYSHAR, J. "Taxation with Costly Administration." Scandinavian Journal of Economics, Vol.93 (1991), pp.75-88.

[17] SLEMROD, J. AND YITZHAKI, S. "Tax Avoidance, Evasion and Administration." NBER working paper 7473 (2000).

[18] YITZHAKI, S. "A Note on Income Tax Evasion: A Theoretical Analysis." Journal of Public Economics, Vol.3 (1974), pp.201-202. 